\title{
PRODUCTION PLANNING AND CONTROL AS ROUTING IN MANUFACTURING NETWORKS
}

\author{
Terje Bach, Bjørn Jæger, Arild Hoff \\ Molde University College, Specialized University in Logistics \\ P.O.Box 2110, 6402 Molde \\ Norway \\ terje.bach@himolde.no,bjorn.jager@himolde.no, arild.hoff@himolde.no
}

\begin{abstract}
In this paper, we explore the use of network routing for production planning and control in manufacturing of complex industrial products. Such a product is the result of a joint effort of many manufacturing companies; each considered a collection of work centre nodes connected by transportation links forming a company-wide manufacturing network. Each company is, in turn considered a manufacturing node connected to other manufacturers by transportation links forming a distributed manufacturing network that produces the final product. We model the manufacturing network as a distribution network where the incoming and outgoing inventories of products are distinct nodes in addition to the work centre nodes. Production planning and control are done by minimizing the cost of handling all products in all work centre nodes. This formulation provides a scalable and flexible production planning and control scheme adhering to the networked structure of manufacturing of complex products. We apply the model to a company-wide manufacturing network as a first approach. A case study using the model demonstrates production planning using network routing at a manufacturer of ship propulsion engines.
\end{abstract}

Keywords: Discrete manufacturing, next generation manufacturing systems, industry 4.0, advanced manufacturing, smart manufacturing

\section{Introduction}

Contemporary manufacturing industries relay on top-down production planning and control (PPC) executed via enterprise resource planning (ERP) systems possibly connected via various intermediate systems to the shop floor [1]. This vertical planning and control mechanism has served manufacturing plants well, especially within mass production industries. As the demand turns toward customized products, manufacturing needs to change their processes to incorporate production techniques such as postponement, agile and flexible manufacturing to satisfy their customers [2]. The proven PPC mechanisms, like standardization and lean, become less suitable while more flexible and dynamic mechanisms are called for. The general network paradigm offers flexible reconfigurable systems that can handle unstable and changing customer demands. We see the move towards networks by the increased focus on collaboration within integrated manufacturing networks $[3,4]$. Such manufacturing systems allow rapid adjustments according to their production capacity and functionality needed especially by customer-centric manufacturers as engineer-to-order companies producing customer specific products $[5,6]$. Historically, a single company executed the major portion of manufacturing operations needed to create a final product [7]. Over the years, companies found it cost efficient to specialize on core competencies while outsourcing supporting activities, and thus adding an increasing number of connections, resulting in a global network of manufacturers. Specialization on core activities followed by outsourcing of support activities have in effect turned manufacturers into assemblers since the major part of their product comes from suppliers, as can be seen from the large portion of the value creation that goes to purchasing. In the EU in 2015, the 2.1 million enterprises classified as manufacturers, spent in average $74 \%$ of their turnover on purchases of goods and services [8]. For manufacturers of highly complex specialized engineer-to-order products like ships, the procurement costs are typically around $80 \%$ of the total costs [9]. Generally, we recognize this as the 80/20 Pareto principle for all companies in the manufacturing sector resulting in $80 \%$ assembly and $20 \%$ physical transformation of materials or components. We see that the total manufacturing effort for a complex industrial product has become an assembly process involving a network of manufacturers. This is illustrated in Figure 1 in which the square blue node represents a manufacturer with four first-tier suppliers, of which each have subsuppliers etc. The blue node is itself a first-tier supplier of two other manufacturers. 


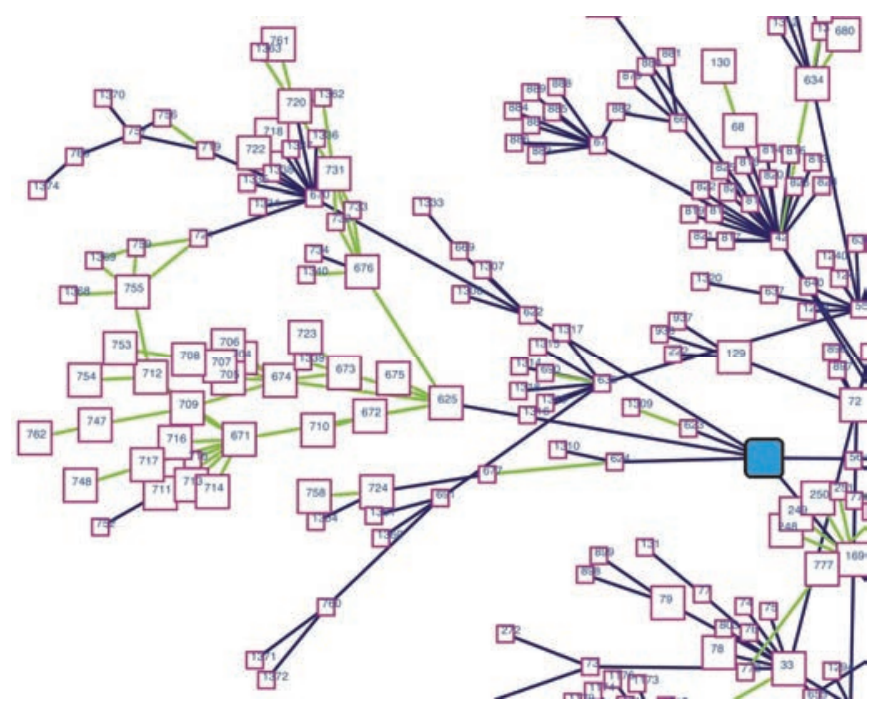

Figure 1: General manufacturing network structure. Each node is a manufacturing company contributing to in average around $20 \%$ of the physical transformation of a product. Adapted from [10].

The efficient operation of such a distributed manufacturing network rely largely on the internet as a communication platform. Internet, a global distributed network, developed from first providing links between networks (i.e. inter-net), becomes a network-of-network nodes in which each node runs the entire IP-communication protocol stack. The Internet platform supports virtually all data communication needs. From local communication between tiny internet-enabledobjects (Internet-of-Things) in Cyber-Physical-Systems, to global communication in global production networks [11]. The global infrastructure of the internet provides a ubiquitous communication service platform available locally within manufacturers and within the global manufacturing network. The map of major Internet Service Providers (ISPs) in Figure 2 illustrates the ubiquitous global coverage of the internet platform. The granularity of internet-connected nodes is even finer than shown in Figure 2 since each ISP controls many IP-nodes. Thus, the internet forms a backbone onto which manufacturing networks operates, as described by e.g. the Digital Manufacturing Platforms for Connected Smart Factories reported by the European Commission [12].

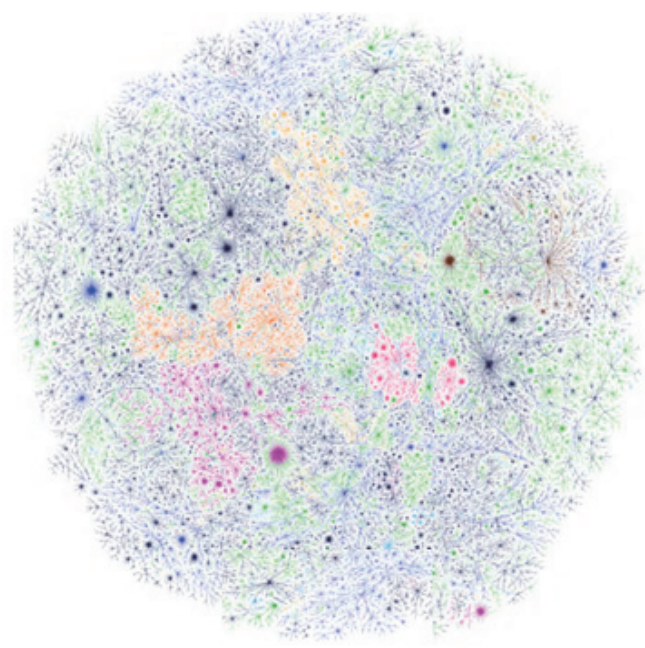

Figure 2: Map of the internet showing major ISP's. Adapted from [10].

In such a network environment, the traditional PPC regime of shop floors is less suitable. We propose to use network management algorithms to plan and control the manufacturing resources in nodes and the timing between nodes in order to transform raw materials into final products across a manufacturing network. In particular, and as a first approach, we do the routing part using distribution network routing. Within a manufacturing company, shop floor operations of grinding, milling, painting, etc. are executed in work centres connected by transportation systems like conveyor belts moving the semi-finished goods along the route through the shop floor. Modelling this as a network, allows us to move from the traditional PPC mechanisms to apply network management algorithms for PPC. By this, there are two alternative ways to handle PPC, one traditional shop floor regime of scheduling operations for each work centre, and another new approach by network routing algorithms for PPC. Below, we summarize the main characteristics of the two regimes. 
- Characteristics of traditional shop floor PPC scheduling following either the push or the pull style:

o Push-style: Plan utilization of available resources and release the material to the production line.

- Hard to handle congestion

- Supports shifts in demand

- Hard to handle errors

- Hard to handle scaling, especially in distributed manufacturing networks

o Pull-style: Control the release of the material by considering the status of work centres.

- Supports congestion avoidance

- Hard to handle shifts in production level

- Hard to handle errors

- Hard to handle scaling, especially in distributed manufacturing networks

- Characteristics of a manufacturing network PPC:

o Routing-style: resources allocated along routes in the network onto which jobs are released if there is enough capacity.

- Supports congestion avoidance

- Supports shifts in demand

- Supports dynamic reconfiguration by rerouting

- Supports scaling across both in-house and inter-company manufacturing networks

We see that a manufacturing network PPC regime provides a potential to handle congestion, shifts in demand, reconfiguration and scaling in a better manner than the traditional shop floor PPC.

\section{The Network Paradigm in Manufacturing}

Manufacturing networks defined from a societal perspective describing manufacturers is a cluster of companies, for example [13]: "A modern manufacturing network is composed of cooperating OEM plants, suppliers and dealers that produce and deliver final products to the market." Research on manufacturing networks has also appeared within operational management, starting within PPC in a single company for [14, 15, 16, 17]. Exemplified by [14]: "By manufacturing network we mean the plant or plants of the manufacturing firm and the relationships with external suppliers." Multi-company manufacturing networks have gained attention, e.g. with work comparing operations of the internal manufacturing network with operations in the extended supply chain [18]. Within Industry 4.0, the combined efforts in an extended value chain is recognized. The horizontal integration in collaborative manufacturing networks balance risks while combining resources to expand market opportunities, and the companies can utilize available capacities more efficiently [2]. Figure 3 illustrates the development of manufacturing paradigms into manufacturing networks.

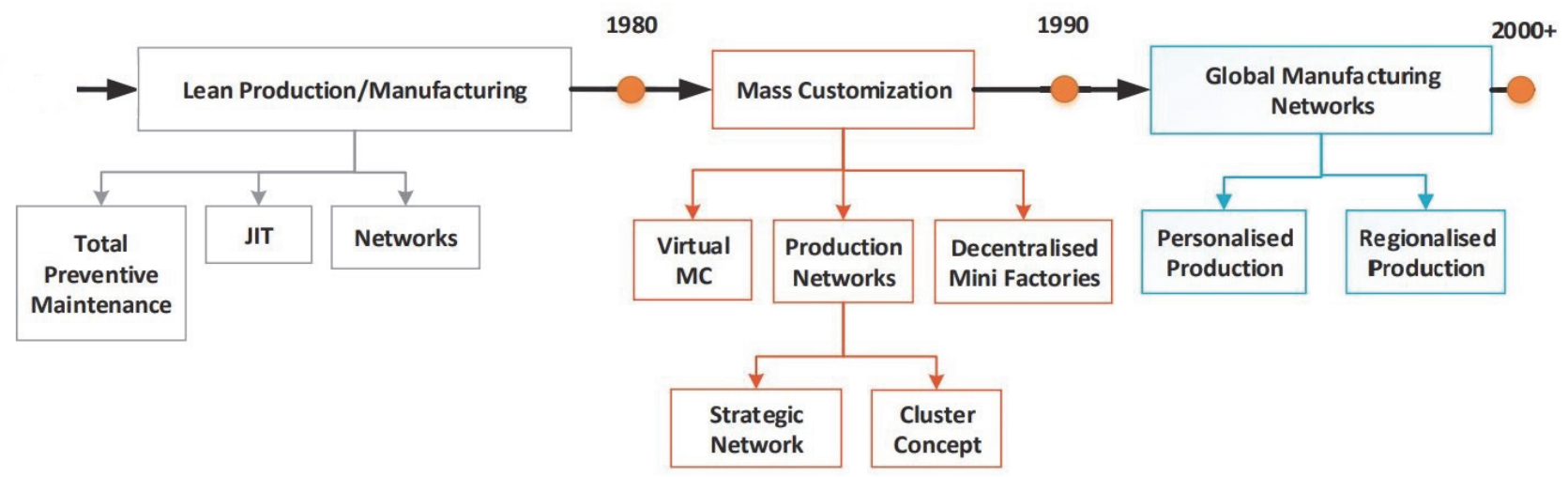

Figure 3: Evolution of Manufacturing Paradigms. Adapted from [13].

In the literature, we find little or no attention paid to the end-to-end PPC across the complete manufacturing network involved in making a single product. In light of the 80/20 Pareto distribution of external to internal operations, we look at manufacturing as an inherent distributed network in which network algorithms handles PPC routing. In doing so we define a manufacturing network as a composition of cooperating manufacturing nodes that produce final products to the market. This definition fits various levels of abstraction, from a network within a single machine, within a site, to within a global manufacturing network. 


\section{A Case Study: Network Routing at a Manufacturer of Ship Propulsion Engines}

We studied a manufacturing company in order to get deeper insight in how routing is done in practice. The company is representative for medium sized companies regarding manufacturing processes, manufacturing environment, and competence. The company delivers systems to the maritime industry regionally and globally. They position themselves in the high-end segment of these markets, delivering technically advanced products with high quality. The production is a combination of make-to-order (MTO) and engineer-to-order (ETO), by engineering the product to some extent for production according to the specific customer's needs. According to the CEO their production process can be considered as a standardized MTO process for around $80-90 \%$ of the manufactured product, leaving only $10-20 \%$ for ETO. However, the numbers are opposite when considering what constitutes their competitive power. I.e. $80-90 \%$ of their competitive power comes from the 10-20\% ETO segment supporting flexibility, short delivery time, and frequent changes of specifications during the manufacturing process. An additional factor observed is that over the last years, the after-market and services have increased in importance. Hence, the need for alternative routes for rerouting to gain flexibility is considered as high. Figure 4 shows the layout of the shop floor at the case company. Their layout organize work centres with respect to their functions and not based on the sequence of operations [19].

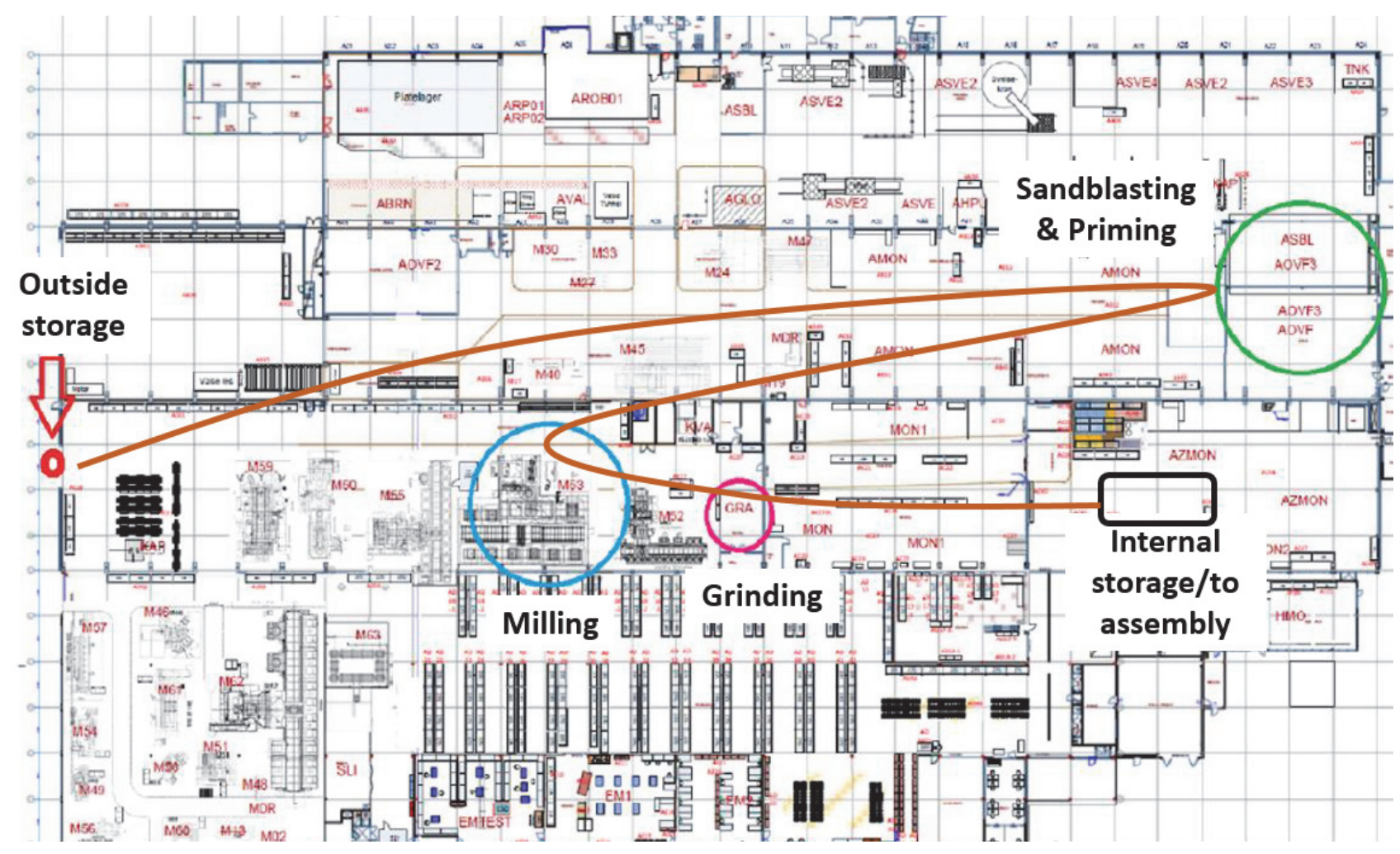

Figure 4: Shop floor at the case company showing a route from outside stock, through sandblasting and priming, milling, grinding and to an internal storage before assembly work centres.

At the case company, many products have at least one alternative route, but for some large complex products, there is only one route. Currently, the routing at the case company is configured in the ERP system. To illustrate, for the Gear axle product $\left(\mathrm{P}_{1}\right)$, two possible routes are available. Route one consist of 60 minutes processing at Work Centre M57, followed by 30 minutes processing at Work Centre M60. The alternative Route two consists of 100 minutes processing time at Work Centre M54, followed by 20 minutes processing at Work Centre M47. Processing times at nodes includes necessary set-up time. If route one is not available then route two is the only existing alternative for this product. Figure 5 shows a graphical representation of the two different routes for the Gear axle.

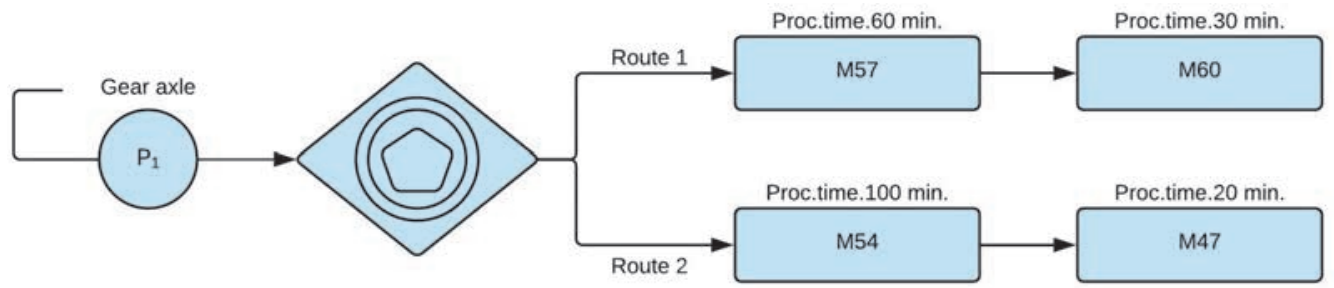

Figure 5: Two different routes for the Gear axle product. 
Managers on the shop floor typically decide to reroute in case problems occur or if they see the need for moving products from one work centre to another.

Another example involves routing via an external subassembly manufacturer. The product Rotor part $\left(\mathrm{P}_{2}\right)$ have three alternative routes. Route 1 is in-house production with 60 minutes processing time in Work Centre M54, followed by 30 minutes processing in Work Centre M60. The alternative route 2 consist of 80 minutes processing time in Work Centre M57, followed by 30 minutes processing in Work Centre M60. Route 3 is outsourcing the production to an external manufacturer "Outsourced", followed by 15 minutes internal quality control. Figure 6 shows a graphical representation of the three different routes for the Rotor part product.

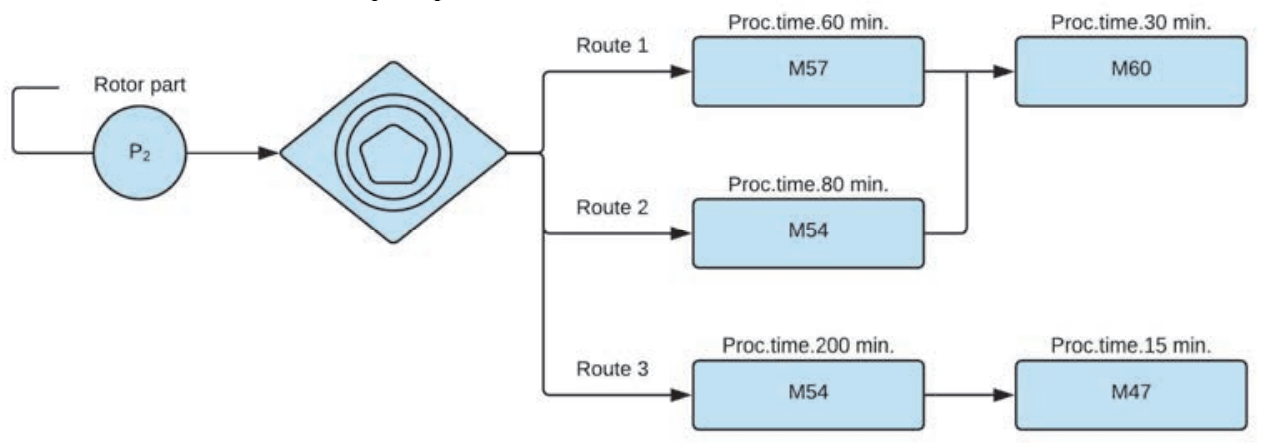

Figure 6: Example of routing alternatives

\section{A Manufacturing Network Model for Planning and Control}

We can model this shop-floor problem as a distribution network defining the incoming and the outgoing inventories of products as separate nodes in the same manner as the work centre nodes. Parameters and variables are defined as:

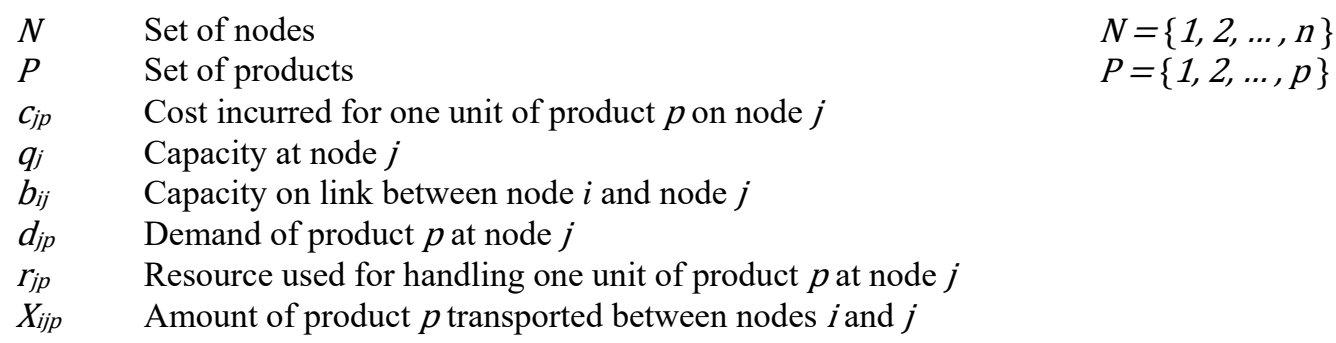

The network is defined as $(N \times N \times P)$ with links of all products between all node combinations. However, not all connections should be possible, and these are defined with zero capacity to prevent any transport on the links. Hence, the mathematical model has the following form:

$$
\operatorname{minimize} \sum_{p \in P} \sum_{j \in N} c_{j p} \sum_{i \in N} X_{i j p}
$$

Such that

$$
\begin{array}{ll}
\sum_{i \in N} X_{i j p}-\sum_{k \in N} X_{j k p}=d_{j p} & \forall j \in N, \forall p \in P \\
\sum_{p \in P} \sum_{i \in N} r_{j p} X_{i j p} \leq q_{j} & \forall j \in N \\
\sum_{p \in P} X_{i j p} \leq b_{i j} & \forall i, j \in N \\
X_{i j p} \geq 0 \text { and integer } & \forall i, j \in N, \forall p \in P
\end{array}
$$

The objective function (1) states that we should minimize the cost of handling all products in all nodes. The cost can be related to the resource used $\left(r_{j p}\right)$, but it can also include other elements like use of raw materials, products and other commodities. Due to the diversity of machines, such costs could be different for handling the various products in different nodes. 
Constraints (2) specify that for each product, the difference between the flow into a node and the flow out of the same node, should meet the demand of the node. The demand is defined as a negative value (number of units) at the incoming inventory nodes, and a positive value (the same number of units) at the outgoing inventory nodes. At the workstation nodes, the demand is defined as zero, ensuring a balance of products sent in to and out from the node.

Constraints (3) state that the capacity at each node should not be exceeded, while constraints (4) ensure that the capacity at the links is met. Impossible links are defined with zero capacity, preventing any transport on these, while the possible links are defined with a capacity sufficiently high. At last, constraints (5) ensure non-negativity and integer on all $X$-variables.

\section{Application of the Model at the Case Company}

Figure 7 shows the generic layered network for the production of the example products Gear axle $\left(\mathrm{P}_{1}\right)$ and Rotor part $\left(\mathrm{P}_{2}\right)$. The leftmost nodes 1 and 2 represent the starting states, labelled staging. The rightmost nodes 9 and 10 represent end states of the products, labelled Storage. No processing takes place within these start and end nodes. The inner nodes 3 through 8 represent work centres, performing some form of processing or transformation of the products. The resource centres can, in principle, be either internal or external to the company. The model can easily be scaled to handle any number of work centres and products, both within one company, but also including several companies in a network. However, for large networks, there might be a problem to find the optimal solution by exact optimization methods. In such cases, heuristics can be designed to identify good solutions as shown in [20] for a related routing problem.

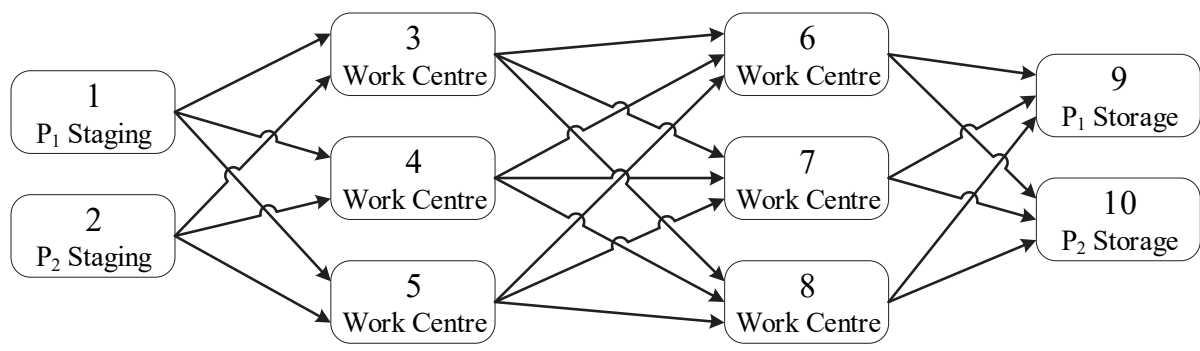

Figure 7: Generic layered network model for the production of two products, $\mathrm{P}_{1}$ and $\mathrm{P}_{2}$.

From the defined routes in Figures 5 and 6, it is clear that not all connections in Figure 7 are relevant for the two example products. There are two routes for the Gear axle product $\mathrm{P}_{1}$, and three routes for the Rotor part product $\mathrm{P}_{2}$. Figure 8 shows the network model of the possible routes for the example products.

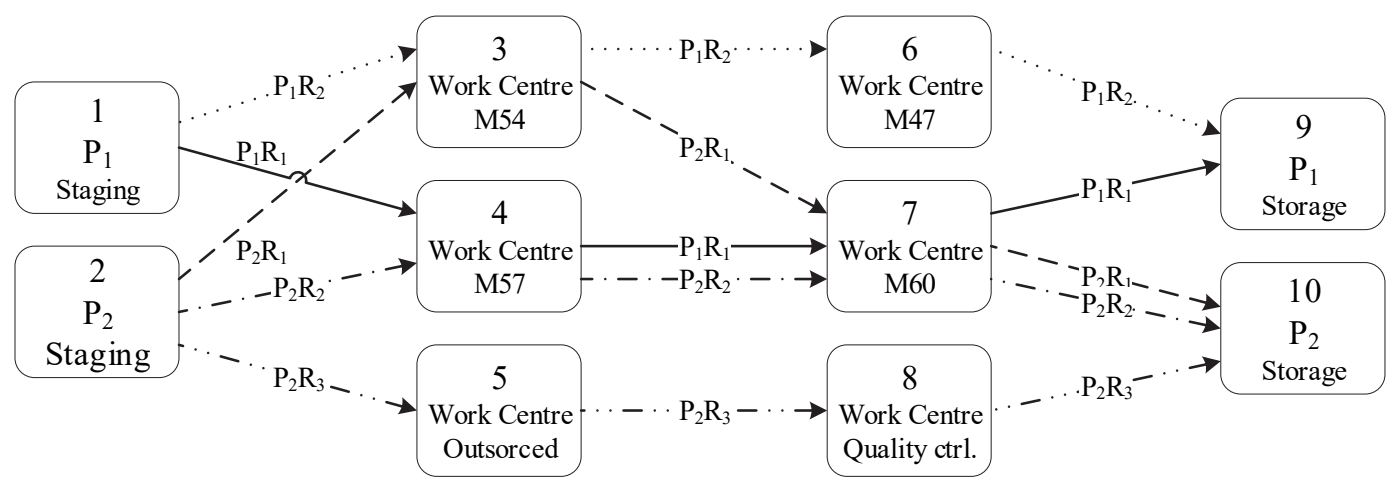

Figure 8: Network model of the alternative product routes for the case company. The individual routes are labelled with product- and route number.

\section{Numerical Setup}

Table 1 lists work centres processing times in minutes for each product as deducted from Figure 5 and 6 . Work centres are operating for 8 hours daily; hence, the capacity over the eight-hour planning horizon is 480 minutes for each work centre. 
Table 1: Work centres processing times in minutes for products

\begin{tabular}{|l|c|c|c|c|c|c|}
\hline & \multicolumn{6}{|c|}{ Work Centre Nodes } \\
\hline Product & 3 & 4 & 5 & 6 & 7 & 8 \\
\hline Gear axle $\left(\mathrm{P}_{1}\right)$ & 100 & 60 & - & 20 & 30 & - \\
\hline Rotor part $\left(\mathrm{P}_{2}\right)$ & 60 & 80 & 200 & - & 30 & 15 \\
\hline
\end{tabular}

Table 2 summarises the possible production routes together with the route cost expressed as total processing times in minutes for each combination of product and route. For each product, routes are numbered from lowest to highest processing time.

Table 2: Summary of routes and costs. "X" means node is part of route, "-" indicates not part of route.

\begin{tabular}{|c|c|c|c|c|c|c|c|c|c|c|c|}
\hline Route & \multicolumn{10}{|c|}{ Nodes } & Route cost \\
\hline & 1 & 2 & 3 & 4 & 5 & 6 & 7 & 8 & 9 & 10 & \\
\hline $\mathrm{P}_{1} \mathrm{R}_{1}$ & $\mathrm{X}$ & - & - & $\mathrm{X}$ & - & - & $\mathrm{X}$ & - & $\mathrm{X}$ & - & 90 \\
\hline $\mathrm{P}_{1} \mathrm{R}_{2}$ & $\mathrm{X}$ & - & $\mathrm{X}$ & - & - & $\mathrm{X}$ & - & - & $\mathrm{X}$ & - & 120 \\
\hline $\mathrm{P}_{2} \mathrm{R}_{1}$ & - & $\mathrm{X}$ & $\mathrm{X}$ & - & - & - & $\mathrm{X}$ & - & - & $\mathrm{X}$ & 90 \\
\hline $\mathrm{P}_{2} \mathrm{R}_{2}$ & - & $\mathrm{X}$ & & $\mathrm{X}$ & - & - & $\mathrm{X}$ & - & - & $\mathrm{X}$ & 110 \\
\hline $\mathrm{P}_{2} \mathrm{R}_{3}$ & - & $\mathrm{X}$ & - & - & $\mathrm{X}$ & - & - & $\mathrm{X}$ & - & $\mathrm{X}$ & 215 \\
\hline
\end{tabular}

Node cost $c_{j p}$ equals resource usage $r_{j p}$ in this example. However, resource usage might be any arbitrary function reflecting the value of the resources consumed. Furthermore, link capacities $b_{i j}$, are equal to a sufficiently large value for all links participating in a route. Hence, link capacities do not restrict flow in this example case.

\section{Analysis}

The distribution network was modelled in Excel and solved using Frontline's Analytic Solver Platform for three scenarios, representing low, medium and high demand for the product $\mathrm{P}_{2}$, while the demand for product $\mathrm{P}_{1}$ is kept constant at 5 units in all scenarios. When demand is low $\left(\mathrm{P}_{1}=5\right.$ and $\left.\mathrm{P}_{2}=7\right)$ all demand is processed along the primary route for both products. For $\mathrm{P}_{1}$ this means that the demanded quantity of 5 units follows route $\mathrm{P}_{1} \mathrm{R}_{1}$ through nodes 1-4-7-9. Similarly, for $\mathrm{P}_{2}$ the selected route $P_{2} R_{1}$ is $2-3-7-10$ for all 7 units.

When demand is medium $\left(\mathrm{P}_{1}=5\right.$ and $\left.\mathrm{P}_{2}=10\right)$ the demand for $\mathrm{P}_{1}$ continues to use the same route as before. However, the processing of $\mathrm{P}_{2}$ is now distributed between two alternative routes. A number of 8 units along its route $\mathrm{P}_{2} R_{1}$ through nodes 2-3-7-10 as before, and a number of 2 following its route $\mathrm{P}_{2} \mathrm{R}_{2}$ through nodes 2-4-7-10.

Finally, when the demand increases to high $\left(\mathrm{P}_{1}=5\right.$ and $\left.\mathrm{P}_{2}=12\right)$ the demand and route for $\mathrm{P}_{1}$ continues to be the same as before. However, the processing of $\mathrm{P}_{2}$ is now distributed between three alternative routes, of which one goes through an external supplier. 8 units follows its route $\mathrm{P}_{2} \mathrm{R}_{1}$ through nodes 2-3-7-10, 2 units follows route $\mathrm{P}_{2} \mathrm{R}_{2}$ through nodes 2-47-10, and 2 units follows route $\mathrm{P}_{2} \mathrm{R}_{3}$ through nodes 2-5-8-10.

From the above scenarios, it is evident that as long as the demand for both products is low, default routes are used for both products. When demand increases beyond the capacities of the default routes, alternative routes are being selected, allowing utilization of unused production capacity.

Table 3: Available and used processing capacity for the Gear axle and Rotor part

\begin{tabular}{|l|c|c|c|c|c|c|}
\hline & \multicolumn{7}{|c|}{ Work centre nodes } \\
\hline & 3 & 4 & 5 & 6 & 7 & 8 \\
\hline Available capacity & 480 & 480 & 480 & 480 & 480 & 480 \\
\hline Used capacity-low demand $\left(\mathrm{P}_{1}=5\right.$ and $\left.\mathrm{P}_{2}=7\right)$ & 420 & 300 & 0 & 0 & 360 & 0 \\
\hline Used capacity-medium demand $\left(\mathrm{P}_{1}=5\right.$ and $\left.\mathrm{P}_{2}=10\right)$ & 480 & 460 & 0 & 0 & 450 & 0 \\
\hline Used capacity-high demand $\left(\mathrm{P}_{1}=5\right.$ and $\left.\mathrm{P}_{2}=12\right)$ & 480 & 460 & 400 & 0 & 450 & 30 \\
\hline
\end{tabular}

\section{Conclusion}

We have proposed a network routing model for production planning and control in manufacturing of complex industrial products. Using a network model paradigm provides a potential for more flexible and robust production, improved 
utilization of capacities, shorter lead times, improved overview of facilities usage and unused capacity. We developed a distribution network model of the manufacturing network where the routing part of production planning and control were done by minimizing the cost of handling products by work centre nodes. We demonstrated the applicability of the model by a case study of a manufacturer of ship propulsion engines in which we found routes for the production of two products. This network model paradigm for ETO/MTO production is a step on the path towards a future with decentralized dynamic routes of Industry 4.0.

Acknowledgement: This research was supported by the Norwegian Research Council grant Manufacturing Network 4.0. We thank the case company for valuable information, as well as master students Halvor F. Andreassen, Ingrid Låtun, Marius Ullaland and Christian Skuterud of Molde University College for their support of data from the shop floor.

\section{References}

[1] Aslan, B., Stevenson, M., Hendry, L. C.: The applicability and impact of Enterprise Resource Planning (ERP) systems: Results from a mixed method study on Make-To-Order (MTO) companies. Computers in Industry, 70, 127$143(2015)$.

[2] Brettel, M., Friederichsen, N., Keller, M., Rosenberg, M.: How virtualization, decentralization and network building change the manufacturing landscape: An Industry 4.0 Perspective. International Journal of Mechanical, Industrial Science and Engineering, 8(1), 37-44 (2014).

[3] Boccaletti, S., Bianconi, G., Criado, R., Del Genio, C. I., Gómez-Gardenes, J., Romance, M., Sendiña-Nadal, I., Wang, Z., Zanin, M.: The structure and dynamics of multilayer networks. Physics Reports, 544(1), 1-122 (2014).

[4] Bortolini, M., Galizia, F. G., Mora, C.: Reconfigurable manufacturing systems: Literature review and research trend. Journal of Manufacturing Systems, 49, 93-106 (2018).

[5] Kjersem, K., Halse, L. L., Kiekebos, P., Emblemsvåg, J.: Implementing lean in engineer-to-order industry: a case study. In: IFIP International Conference on Advances in Production Management Systems (pp. 248-255). Springer, Cham. (September 2015).

[6] Helo, P., Suorsa, M., Hao, Y., Anussornnitisarn, P.: Toward a cloud-based manufacturing execution system for distributed manufacturing. Computers in Industry, 65(4), 646-656 (2014).

[7] Magal, S. R., Word, J.: Essentials of business processes and information systems. Wiley Publishing. (2009).

[8] Eurostat/European Commission: Statistics for the European Union's (EU) manufacturing sector. Annual detailed enterprise statistics for industry. Retrieved from https://ec.europa.eu/eurostat/statisticsexplained/index.php/Manufacturing_statistics_-_NACE_Rev._2\#Structural_profile (last modified 2015).

[9] Giskeødegård, M. F., Guvåg, B.: 18 Nærhet og Avstand. Book chapter in Det regionale i det internasjonale: Fjordantologien. Kunnskapsforlaget. 333-354 (2018).

[10] Sayama, H., Cramer, C., Sheetz, L., Uzzo, S.: NetSciEd: Network Science and Education for the Interconnected World. arXiv preprint arXiv:1706.00115 (2017).

[11] Coe, N. M., Dicken, P., Hess, M.: Global production networks: realizing the potential. Journal of economic geography, 8(3), 271-295 (2008).

[12] European Commission, Work Programme 2018-2019. DT-ICT-07-2018-2019: Digital Manufacturing Platforms for http://ec.europa.eu/research/participants/portal/desktop/en/opportunities/h2020/topics/dt-ict-07-2018-019.html

[13] Mourtzis, D., Doukas, M.: Design and planning of manufacturing networks for mass customisation and personalisation: challenges and outlook. Procedia CIRP, 19, 1-13 (2014).

[14] Feldmann, A., Olhager, J., Persson, F.: Designing manufacturing networks - an empirical study. In: Advances in production management systems, pp. 95-102. Springer, Boston, MA (2007).

[15] Skinner, W.: Manufacturing-missing link in corporate strategy. Harvard Business Review, (May 1969).

[16] Wang, S., Wan, J., Li, D., Zhang, C.: Implementing smart factory of industrie 4.0: an outlook. International Journal of Distributed Sensor Networks, 12(1), 3159805 (2016).

[17] Haskose, A., Kingsman, B. G., Worthington, D.: Performance analysis of make-to-order manufacturing systems under different workload control regimes. International journal of production economics, 90(2), 169-186 (2004).

[18] Rudberg, M., Olhager, J.: Manufacturing networks and supply chains: an operations strategy perspective. Omega, 31(1), 29-39 (2003).

[19] Andreassen, H. F., Låtun, I.: Lead time reduction and the correlation between delays of components and final products in ETO manufacturing companies within the scope of Industry 4.0; a case study. Master's degree thesis, Molde University College (2018).

[20] Costa, M.-C., Hertz, A., Mittaz, M.: Bounds and Heuristics for the Shortest Capacitated Paths Problem. Journal of Heuristics, 8, 449-465 (2002). 